\title{
Amorphastilbol exerts beneficial effects on glucose and lipid metabolism in mice consuming a high-fat-diet
}

\author{
WOOJUNG LEE ${ }^{1}$, JUNGYEOB HAM ${ }^{1}$, HAK CHEOL KWON ${ }^{1}$, GOO YOON $^{2}$, \\ GYU-UN BAE ${ }^{3}$, YONG KEE KIM ${ }^{3}$ and SU-NAM KIM ${ }^{1}$
}

\author{
${ }^{1}$ Natural Medicine Center, KIST Gangneung Institute, Gangneung, Gangwon 210-340; ${ }^{2}$ College of Pharmacy and \\ Natural Medicine Research Institute, Mokpo National University, Jeonnam 534-729; ${ }^{3}$ Research Center for Cell Fate Control, \\ College of Pharmacy, Sookmyung Women's University, Seoul 140-742, Republic of Korea
}

Received November 26, 2014; Accepted May 21, 2015

DOI: $10.3892 /$ ijmm.2015.2227

\begin{abstract}
In the present study, the anti-diabetic effects of amorphastilbol (APH) from Amorpha fruticosa (AF) were evaluated in high-fat-diet (HFD) mice. HFD-induced blood glucose and insulin levels are significantly reduced in AF extract or APH treatment groups. HFD-induced weight gain was reduced by AF treatment, which is accompanied by reduction of fat mass and adipocyte size and number in white adipose tissues. Furthermore, total cholesterol and low-density lipoprotein-cholesterol levels are decreased in AF- or APH-treated mice. In addition, AF and APH are able to improve insulin sensitivity through inhibition of protein tyrosine phosphatase $1 \mathrm{~B}$, a negative regulator of the insulin-signaling pathway. Taken together, the data suggest that AF has beneficial effects on glucose and lipid metabolism and its pharmacological effects are driven, in part, by its active component, APH. Therefore, AF and APH can be used as potential therapeutic agents against type 2 diabetes and associated metabolic disorders, including obesity, by enhancing glucose and lipid metabolism.
\end{abstract}

\section{Introduction}

Type 2 diabetes mellitus (T2DM) is characterized by the resistance of target tissues to insulin stimulation (1), which is usually associated with hyperglycemia, dyslipidemia, obesity, hypertension, fatty liver, atherosclerosis, certain cancers and cardiovascular diseases (2). Insulin resistance occurs when a

Correspondence to: Professor Yong Kee Kim, Research Center for Cell Fate Control, College of Pharmacy, Sookmyung Women's University, 100 Cheongpa-ro 47-gil, Seoul 140-742, Republic of Korea E-mail: yksnbk@sookmyung.ac.kr

Dr Su-Nam Kim, Natural Medicine Center, KIST Gangneung Institute, 290 Daejeon, Gangneung, Gangwon 210-340, Republic of Korea E-mail: snkim@kist.re.kr

Key words: Amorpha fruticosa, amorphastilbol, peroxisome proliferator-activated receptor $\alpha / \gamma$ agonist, protein tyrosine phosphatase $1 \mathrm{~B}$, insulin resistance normal dose of insulin is incapable of eliciting its metabolic responses (3), which is caused by multiple defects in intracellular events including an impairment of the insulin signaling pathway (4-6). T2DM patients also manifest adipocyte resistance to the antilipolytic effects of insulin $(7,8)$.

The peroxisome proliferator-activated receptor (PPARs) family, belonging to the nuclear hormone receptor family, consists of three isoforms, PPAR $\alpha, \operatorname{PPAR} \beta / \delta$ and PPAR $\gamma$ (9). PPAR $\gamma$, mainly expressed in adipose tissue and vascular tissue/macrophages (10), affects various genes involved in lipid and glucose homeostasis. PPAR $\gamma$ agonists increase insulin sensitivity so that they are used for treatment of T2DM. In addition, the PPAR $\gamma$ agonist promotes adipocyte differentiation and controls mobilization of lipid into adipocytes by inducing the expression of such lipid transport genes as adipocyte fatty acid-binding protein $(a P 2)$, thereby reducing lipotoxicity $(11,12)$. However, several concerns, such as the weight gain associated with increased excess fat, arise in PPAR $\gamma$ agonist-treated T2DM patients (13). Accumulating evidence indicates that the activation of PPAR $\alpha$ predominantly expressed in the liver (14), would stimulate lipid consumption by enhancing the expression of fatty acid oxidation genes, resulting in the amelioration of hyperlipidemia. PPAR $\alpha$ agonists have potent effects on the reduction of plasma triglycerides (15). Due to the distinct metabolic effects of PPAR $\alpha$ and PPAR $\gamma$ agonists on insulin sensitivity and lipid metabolism, development of novel drugs has focused on dual PPARs that possess PPAR $\gamma$ and PPAR $\alpha$ activities. It has been proposed that the simultaneous activation of PPAR $\alpha$ and PPAR $\gamma$ would guarantee more desirable effects with alleviated adverse effects (16-18). Numerous PPAR $\alpha / \gamma$ dual agonists have been identified and tested in obese and insulin-resistant individuals; however, the majority of these drugs have shown unexpected side effects, including weight gain, heart failure, renal failure, urinary cancer and anemia $(19,20)$. Therefore, the development of novel PPAR $\alpha / \gamma$ dual agonists with few adverse effects is urgently required.

Recently, there has been a growing interest in the therapeutic use of natural compounds to treat metabolic syndrome as natural compounds may exert their diverse pharmacological properties by interacting with multiple cellular targets. Recently, we reported that amorphastilbol (APH) from Amorpha fruticosa (AF) stimulates transcriptional activities 
of PPAR $\alpha / \gamma$ (21), and improves glucose and lipid metabolisms in the diabetic $d b / d b$ mouse model (22). To support the anti-diabetic effects of APH and AF, the present study evaluated their pharmacological properties in a high-fat-diet (HFD) mouse model and their effects on insulin sensitivity.

\section{Materials and methods}

Cell culture and chemicals. 3T3-L1 and C2C12 cells were obtained from American Type Culture Collection (Manassas, VA, USA) and cultured in Dulbecco's modified Eagle's medium (DMEM; Invitrogen, Carlsbad, CA, USA) supplemented with 10\% fetal bovine serum (FBS; HyClone Laboratories, Logan, UT, USA) and $1 \%$ penicillin/streptomycin (Invitrogen). APH was synthesized by Dr J Ham [Korea Institute of Science and Technology (KIST) Gangneung Institute] for the in vivo study. Rosiglitazone was purchased from Sigma-Aldrich Co. (St. Louis, MO, USA).

Extract of AF. The fruit of AF (300 g) was extracted three times with $95 \%$ ethanol and evaporated under vacuum at $40^{\circ} \mathrm{C}$. The extract $(20 \mathrm{mg} / \mathrm{ml})$ was reconstituted with $0.8 \%$ carboxymethylcellulose solution.

Animal experiments. All the experiments were performed according to the procedures approved by the KIST's Institutional Animal Care and Use Committee. Seven-week-old male C57BL/6 mice were purchased from the Shizuoka Laboratory Animal Center (Shizuoka, Japan). The mice were housed under conditions of $23 \pm 2^{\circ} \mathrm{C}$ and $55 \pm 5 \%$ humidity with standard light cycles (12-h light/dark). The C57BL/6 mice were fed a regular diet (10\% kcal fat; 38057; Purina Mills Inc., Gray Summit, MO, USA) or an HFD (65\% kcal fat; 101556; Dyets Inc., Bethlehem, PA, USA) for 8 weeks. The mice were orally administered $4 \mathrm{mg} / \mathrm{kg}$ rosiglitazone, $200 \mathrm{mg} / \mathrm{kg}$ AF or $20 \mathrm{mg} / \mathrm{kg}$ APH once a day for 8 weeks prior to the gene expression or blood biomarker analyses. Glucose was measured by tail vein bleeds at the indicated time intervals using an Accu-Chek glucometer (Roche Diagnostics GmbH, Mannheim, Germany), and the serum insulin concentrations were determined by the enzyme-linked immunosorbent assay (Shibayagi, Gunma, Japan). At the end of the experimental period, epididymal white tissue/body weight ratios were measured, and blood samples were obtained from the abdominal aorta to determine plasma biomarker concentrations.

Analysis of plasma biomarkers. After the experiment, blood was collected in tubes containing $0.18 \mathrm{M}$ ethylenediaminetetraacetic acid (EDTA) and centrifuged at 5,000 $\mathrm{x}$ g for $5 \mathrm{~min}$ at $4^{\circ} \mathrm{C}$. Following centrifugation, the plasma was separated for the estimation of the total cholesterol, low-density lipoprotein (LDL)-cholesterol, triglycerides and free fatty acids. The total cholesterol levels were measured by enzymatic methods using SICDIA L T-CHO reagents (Eiken Chemical, Tokyo, Japan), and the LDL-cholesterol levels were determined by enzymatic methods using L-Type LDL-C reagents (Wako Pure Chemical Industries, Osaka, Japan). The triglyceride levels were measured by GPO-HMMPS using the SICDIA L TG reagent, and free fatty acids were measured by enzymatic methods using NEFAZYME-S (from Eiken Chemical).
Histology. Tissue samples of epididymal fat pads were fixed with $4 \%$ buffered formalin and embedded in paraffin. Standard sections of 5- $\mu \mathrm{m}$ were cut and stained with hematoxylin and eosin (H\&E), viewed with an optical microscope, and images were captured (final magnification, x100 or x400).

Myotube formation and immunoblotting. $\mathrm{C} 2 \mathrm{C} 12$ myoblast were cultured in DMEM until 90\% confluent. The cells were differentiated into myotubes with DMEM containing $2 \%$ horse serum for 4 days, and were subsequently incubated for $16 \mathrm{~h}$ in DMEM containing $2 \%$ bovine serum albumin and 10\% FBS in the absence or presence of $0.75 \mathrm{mM}$ palmitate to induce insulin resistance. Subsequently, the AF- or APH-treated cells were stimulated with $100 \mathrm{nM}$ insulin for $10 \mathrm{~min}$. Following stimulation, cells were washed twice with phosphate-buffered saline (PBS) and harvested. The following primary antibodies were used: mouse monoclonal IgG anti-Akt (cs9272; 1:1,000), anti-phospho-Ser473 Akt (cs4060; 1:1,000), anti-IR $\beta$ (cs3020; 1:1,000), anti-phospho-Tyr1150/1151IR $\beta$ (cs3024; 1:1,000), anti-GSK3 $\beta$ (cs9832; 1:1,000), and anti-phospho-Ser9 GSK3 $\beta$ (cs9323; 1:1,000) were purchased from Cell Signaling Technology (Beverly, MA, USA); mouse polyclonal IgG anti-protein tyrosine phosphatase 1B (PTP1B) (sc-1718; 1:1,000) was obtained from Santa Cruz Biotechnology, Inc. (Dallas, TX, USA) and mouse monoclonal IgG anti- $\beta$-actin (A1978; 1:3,000) was purchased from Sigma-Aldrich Co.

2-NBDG glucose uptake assay. The myotubes, which were obtained from the above procedures, were stimulated with $100 \mathrm{nM}$ insulin for $1 \mathrm{~h}$. After insulin stimulation, the myotubes were incubated with $50 \mu \mathrm{M} 2-\mathrm{NBDG}$ (Invitrogen) for $15 \mathrm{~min}$ and were subsequently washed with PBS three times to remove free 2-NBDG. The fluorescence intensity of cells containing 2-NBDG was measured on the Infinite M1000 microplate reader (Tecan Group Ltd., Männedorf, Switzerland) with excitation at $485 \mathrm{~nm}$ and emission at $535 \mathrm{~nm}$.

Gene expression analysis. Total RNA was isolated from mouse tissue using the TRIzol reagent (Invitrogen) according to the manufacturer's instructions. The RNA concentration of each sample was determined by spectrophotometry at $260 \mathrm{~nm}$; the integrity of each RNA sample was evaluated using the Agilent 2100 Bioanalyzer (Agilent Technologies, Santa Clara, CA, USA). cDNA synthesis was performed using $1 \mu \mathrm{g}$ of total RNA in $20 \mu 1$ with random primers and Superscript II reverse transcriptase. Quantitative polymerase chain reaction (PCR) analyses were performed with SYBR-Green fluorescent dye using the 7500 Real-Time PCR system. Data analyses were performed using 7500 System SDS software version 1.3.1 (Applied Biosystem, Foster City, CA, USA). The primer sets for glucose-6-phosphatase (G6Pase) were 5'-ATGACTTTGGG ATCCAGTCG-3' and 5'-TGGAACCAGATGGGAAAGAG-3'; UCP3, 5'-ACAAAGGATTTGTGCCCTCC-3' and 5'-CTTG CCTTGTTCAAAACGGA-3'; and GAPDH, 5'-TTGTTGCCAT CAACGACCCC-3' and 5'-GCCGTTGAATTT GCCGTGAG-3'.

PTP1B activity assay. PTP1B was purchased from Biomol Research Laboratories, Inc. (Plymouth Meeting, PA, USA). The substrate, para-nitrophenyl phosphate (pNPP), was obtained from New England Biolabs, Inc. (Beverly, MA, USA). The 
reaction mixture $(100 \mu \mathrm{l})$ containing $20 \mathrm{mM}$ pNPP and $0.05 \mu \mathrm{g}$ PTP1B in a reaction buffer [50 $\mathrm{mM}$ citrate buffer $(\mathrm{pH} 6.0)$, $0.1 \mathrm{M} \mathrm{NaCl}, 1 \mathrm{mM}$ EDTA, and $1 \mathrm{mM}$ dithiothreitol] was incubated at $37^{\circ} \mathrm{C}$ for 30 min with or without the indicated extract or compound. The reaction was quenched by the addition of $10 \mu \mathrm{l}$ of $10 \mathrm{~N} \mathrm{NaOH}$. The amount of resulting $p$-nitrophenol was estimated by measuring the absorbance at $405 \mathrm{~nm}$.

Statistics. The data are expressed as the mean \pm standard deviation. In the animal experiment (Fig. 1), the data are expressed as the means \pm standard error (SE). Differences between the mean values in the two groups were analyzed using one-way analysis of variance. $\mathrm{P}<0.05$ was considered to indicate a statistically significant difference.

\section{Results}

Anti-diabetic and anti-obesity effects of APH in mice fed a $H F D$. APH has been recently reported as a novel PPAR $\alpha / \gamma$ dual agonist, which ameliorates glucose and lipid impairment in $d b / d b$ mice (22). The present study further evaluated the anti-diabetic and anti-obesity effects of APH in the HFD mouse model. Body weight and blood glucose levels were significantly increased in mice fed a HFD compared to mice fed a normal-diet (Fig. 1A and 1B). AF significantly attenuated the weight gain in the mice fed a HFD, whereas the APH treatment showed a weak effect on the HFD-induced weight gain (Fig. 1A). The blood glucose levels in the mice fed a HFD significantly decreased with the AF and APH treatments (Fig. 1B), which are consistent with the results of the AF and APH treatments in the $d b / d b$ mouse model (22). Of note, the plasma insulin levels in the mice fed a HFD increased (Fig. 1C), resulting in insulin resistance; however, this increase in insulin levels significantly reduced by the AF and APH treatment, respectively (Fig. 1C). Subsequently, epididymal fat mass and adipocyte size and number was measured in each groups. Epididymal fat mass was significantly reduced by administration of either AF or APH (Fig. 2A), which was accompanied by reduction of adipocyte size and number in epididymal fat tissues (Fig. 2B-D). All these data support anti-diabetic and anti-obesity effects of AF, which is driven, in part, by APH.

APH improves metabolic markers in the liver, blood and white adipose tissue. As hepatic gluconeogenesis gene expression is markedly increased in diabetic animals and contributes to hyperglycemia, the expression of G6Pase, one of the key enzymes in gluconeogenesis, was measured in the liver tissue of mice fed a HFD. The expression level of G6Pase significantly reduced in AF- and APH-treated mice (Fig. 3A), supporting the anti-diabetic effects of APH. Concomitantly, AF and APH also markedly diminished the plasma cholesterol and LDL cholesterol levels (Fig. 3B and C), indicating that AF and APH restore cholesterol metabolism in animals fed an HFD. The expression level of UCP3 was subsequently analyzed in white adipose tissue, as UCP3 expression is directly upregulated by PPAR $\gamma$ agonists $(23,24)$. APH treatment significantly increased in UCP3 expression in white adipose tissues (Fig. 3D), which may be associated with the increased rate of lipid metabolism. All these data support that APH exerts beneficial effects on glucose and lipid metabolism in mice fed a HFD.
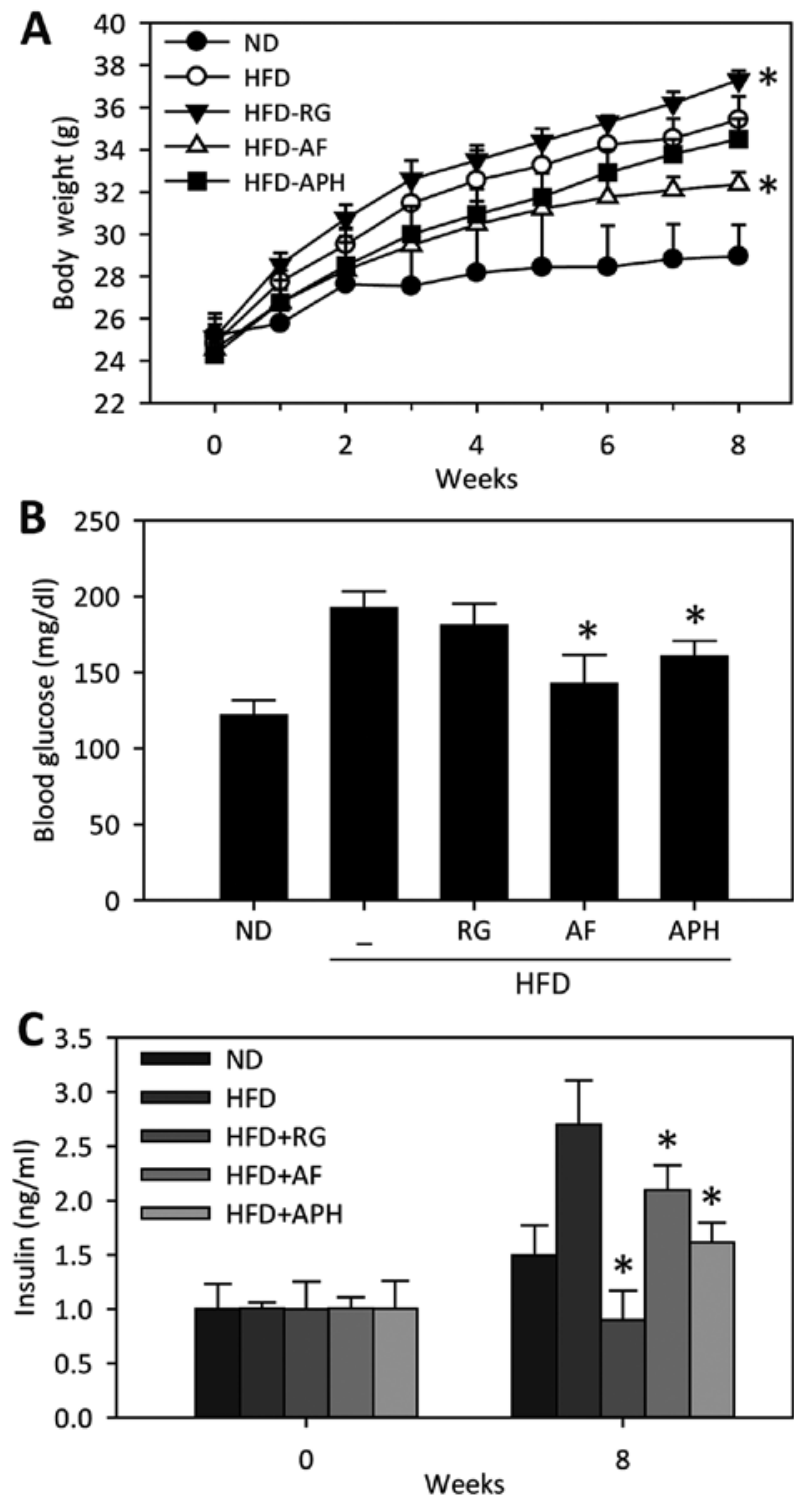

Figure 1. Amorpha fruticosa (AF) and amorphastilbol (APH) improve dyslipidemia and insulin resistance in high-fat-diet (HFD) mice. (A) Whole-body weight changes in rosiglitazone (RG) $(4 \mathrm{mg} / \mathrm{kg})-, \mathrm{AF}(200 \mathrm{mg} / \mathrm{kg})-$, or APH $(20 \mathrm{mg} / \mathrm{kg}$ )-treated mice fed a HFD for 8 weeks ( $\mathrm{n}=7$ for each group). Each bar represents the mean \pm standard error. ${ }^{*} \mathrm{P}<0.05$ vs. HFD control. ND, normal diet. (B) Fasting blood glucose levels of RG- (4 mg/kg), AF- (200 mg/ $\mathrm{kg}$ ) or APH- $(20 \mathrm{mg} / \mathrm{kg}$ ) treated mice fed a HFD ( $\mathrm{n}=7$ for each group) after 8 weeks. (C) Insulin level changes in RG- (4 mg/kg), AF- $(200 \mathrm{mg} / \mathrm{kg})$ or APH- $(20 \mathrm{mg} / \mathrm{kg})$ treated mice fed a HFD at 0 and 8 weeks.

APH improves insulin sensitivity by repressing PTP1B. To further ascertain the positive effects of AF and APH on insulin sensitivity, glucose uptake was first examined in C2C12 myotubes under normal conditions. Glucose uptake in $\mathrm{C} 2 \mathrm{C} 12$ myotubes in the absence or presence of insulin was significantly enhanced by AF and APH treatment under normal conditions (Fig. 4A). Correspondingly, AF and APH application also enhanced the insulin-evoked phosphorylation of IR, Akt and GSK-3 $\beta$ under normal conditions (Fig. 4C). Subsequently, the effects of AF and APH on glucose uptake were investigated under palmitate-induced insulin-resistant conditions. AF and APH also enhanced glucose uptake under insulin-resistant conditions (Fig. 4B). The effects of AF and APH on insulin sensitivity under insulin-resistant conditions 

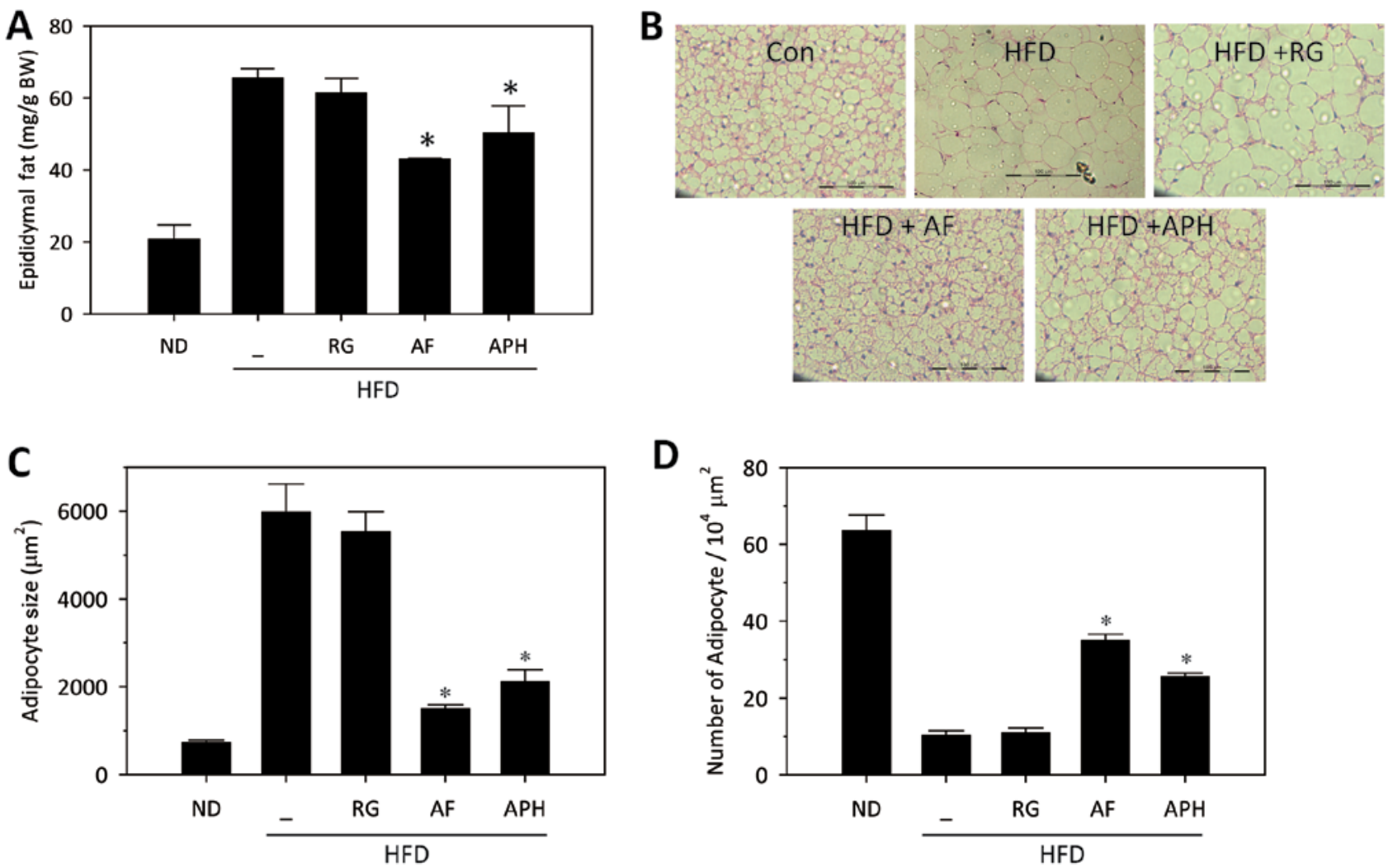

Figure 2. Amorpha fruticosa (AF) and amorphastilbol (APH) reduce fat mass and adipocyte size and number in high-fat-diet (HFD) mice. (A) Average epididymal fat weights of rosiglitazone (RG)-, AF- or APH-treated mice fed a HFD. (B) Hematoxylin and eosin (H\&E) staining of white adipose tissue (WAT) from the mice fed a HFD. Adipocyte (C) size and (D) number were measured from H\&E staining of WAT from the mice fed a HFD.
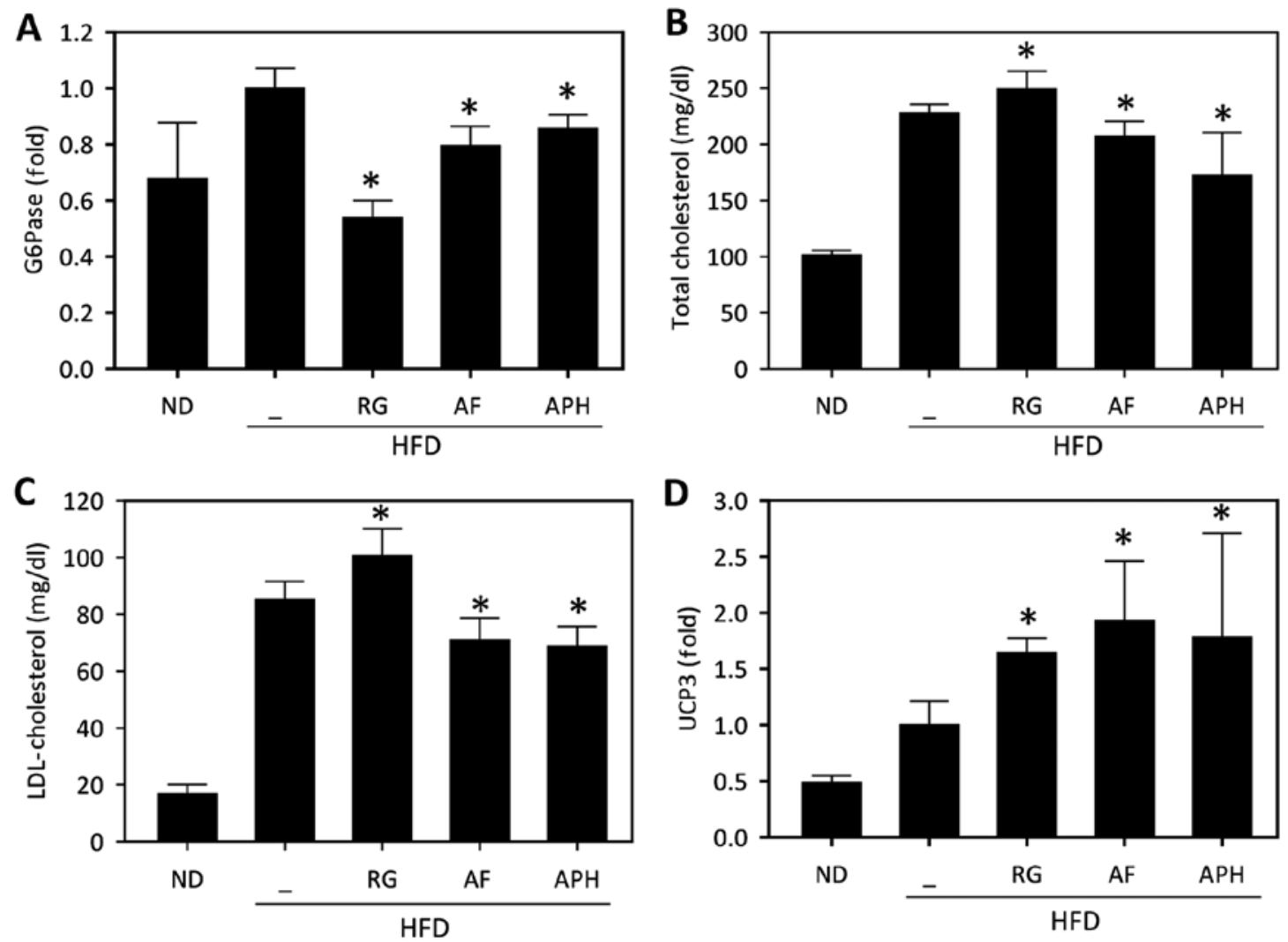

Figure 3. Amorpha fruticosa (AF) and amorphastilbol (APH) improve metabolic markers in the liver, blood and white adipose tissue (WAT). (B) Total cholesterol and (C) LDL-cholesterol levels of rosiglitazone- (RG; $4 \mathrm{mg} / \mathrm{kg})$, AF- (200 mg/kg) or APH- (20 mg/kg) treated high-fat-diet (HFD) mice ( $\mathrm{n}=7$ for each group) after 8 weeks. The relative gene expression levels of (A) G6Pase and (D) UCP3 were determined by quantitative polymerase chain reaction. Data represent the means \pm standard deviation. ${ }^{*} \mathrm{P}<0.05$ vs. HFD control. 
A

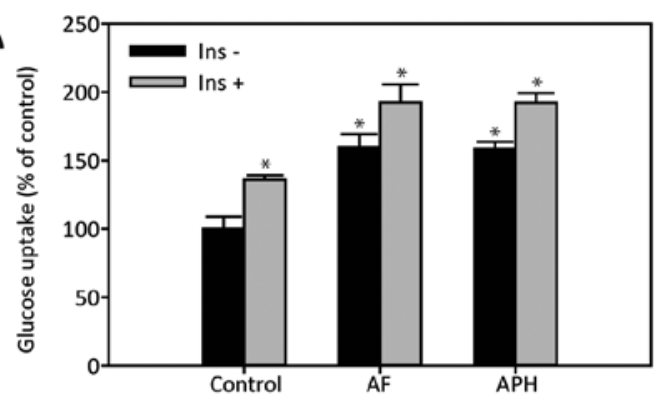

C

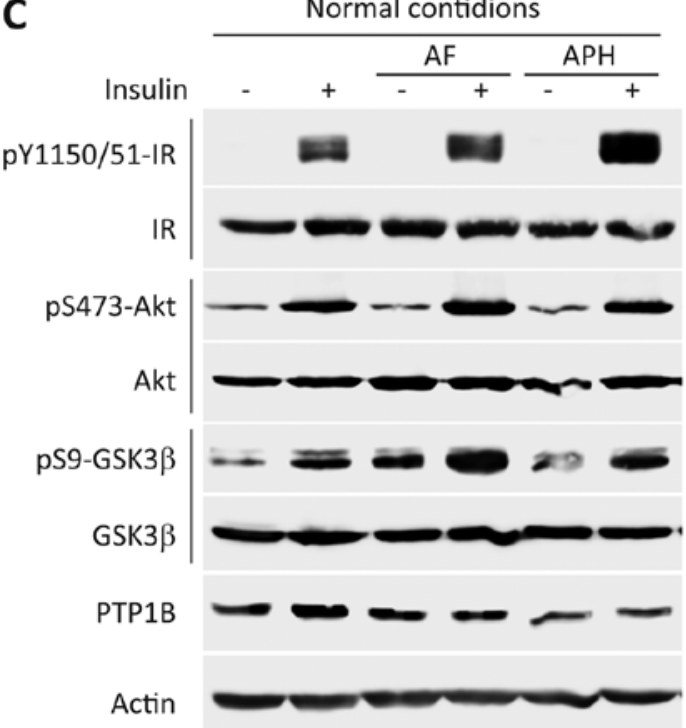

B

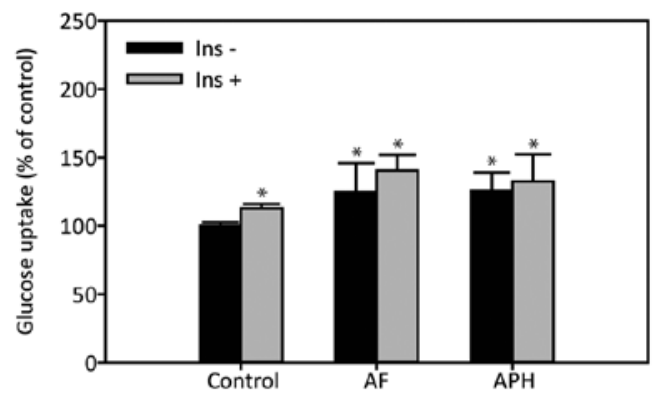

D

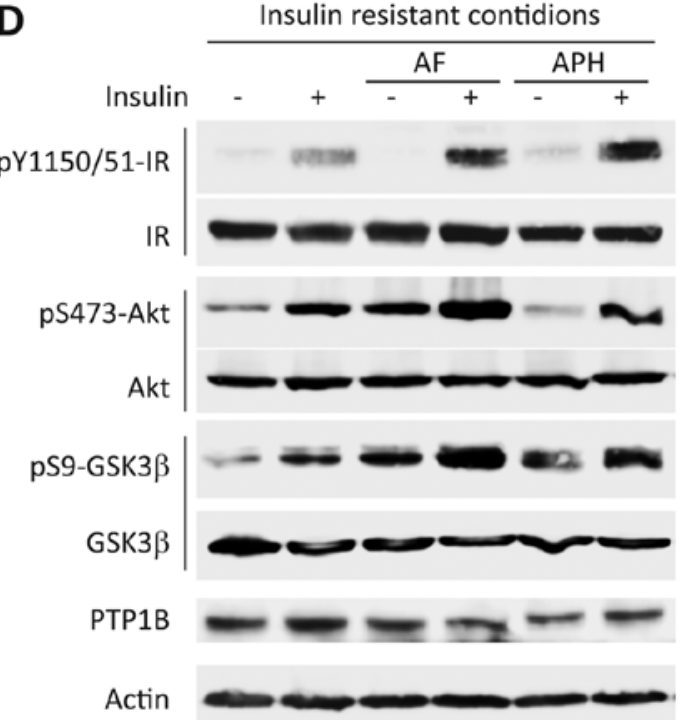

Figure 4. Amorpha fruticosa (AF) and amorphastilbol (APH) enhance insulin sensitivity under normal and insulin-resistant conditions. (A and B) Basal and insulin-stimulated glucose uptake in AF- and APH-treated $\mathrm{C} 2 \mathrm{C} 12$ myotubes under normal and insulin-resistant conditions. Each bar represents the mean \pm standard deviation of triplicate experiments. ${ }^{*} \mathrm{P}<0.05$ vs. cells without any treatment. (C and D) Level of phosphorylation of IR, Akt, GSK-3 $\beta$ and protein tyrosine phosphatase 1B (PTP1B) in AF- and APH-treated C2C12 myotubes under normal and insulin-resistant conditions. Protein levels were analyzed by western blot analysis.

A

\begin{tabular}{ccccc}
\hline & UA & RG & AF & APH \\
\hline IC $_{50}$ & $3.2 \mu \mathrm{M}$ & $21 \mu \mathrm{M}$ & $3.4 \mu \mathrm{g} / \mathrm{ml}$ & $4.2 \mu \mathrm{M}$
\end{tabular}

B Fold: 1 1.1 3.6 2.6 pS473-Akt

Akt

\begin{tabular}{lllll}
1 & 1.1 & 3.6 & 2.6 \\
\cline { 3 - 4 } & & & \\
\hline & & &
\end{tabular}

Fold:

1

1.5 3.3 2.7

pY1150/51-IR

IR

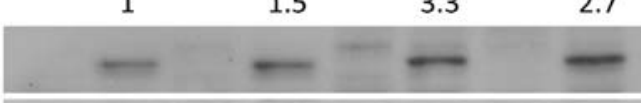

Insulin

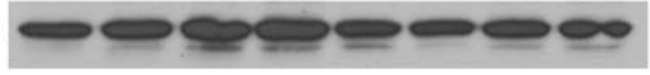

Figure 5. Amorpha fruticosa (AF) and amorphastilbol (APH) improve insulin resistance through protein tyrosine phosphatase 1B (PTP1B) inhibition. (A) The inhibition of PTP1B by ursolic acid (UA), rosiglitazone (RG), $\mathrm{AF}$ or APH was determined as described in Materials and methods. (B) After $1 \mathrm{~h}$ of pretreatment with RG, AF or APH, 3T3-L1 cells were stimulated with insulin $(10 \mu \mathrm{g} / \mathrm{ml})$ for $10 \mathrm{~min}$. The IR $\beta$ tyrosine and Akt phosphorylation levels were determined by immunoblot analysis. were further confirmed by elevated phosphorylation of these downstream proteins in insulin signaling (Fig. 4D). These effects were further confirmed by the observation that insulin activation of the IR-Akt signaling axis was significantly enhanced by AF and APH in 3T3-L1 adipocytes (Fig. 5B). To explore the underlying mechanism responsible for improvement of insulin sensitivity by AF and APH, the expression levels of PTP1B, a well-known negative regulator of the insulin signaling pathway, were determined. AF and APH treatment led to a significant decrease in PTP1B expression in $\mathrm{C} 2 \mathrm{C} 12$ myotubes, indicating that PTP1B may be a potential target of $\mathrm{AF}$ and $\mathrm{APH}$ (Fig. 4C and D). In addition, PTP1B activity was inhibited by AF and $\mathrm{APH}$, with an $\mathrm{IC}_{50}$ of $3.4 \mu \mathrm{g} / \mathrm{ml}$ and $4.2 \mu \mathrm{M}$, respectively, whereas rosiglitazone has a weak effect on PTP1B ( $\left(\mathrm{IC}_{50} 21 \mu \mathrm{M}\right)$ (Fig. 5A). All these results suggest that improvement of insulin sensitivity by AF and APH are, at least in part, mediated by repressing PTP1B.

\section{Discussion}

Numerous oral anti-diabetic agents are currently used for the treatment T2DM patients, such as insulin secretagogues (sulfonylureas and meglitinides), metformin, thiazolidinediones 
(rosiglitazone and pioglitazone), $\alpha$-glucosidase inhibitors and incretin-based therapies (exenatide and sitagliptin). Insulin secretagogues increase insulin secretion from $\beta$ cells through the inhibition of the KATP channel and subsequently, decrease plasma glucose. The incretin-based therapies control post-meal glucose excursions by increasing insulin release and decreasing glucagon secretion, leading to a decrease in plasma glucose $(25,26)$. The anti-diabetic effects of these two drug classes are dependent upon the actions of insulin. By contrast, metformin and thiazolidinediones, which are classified as insulin sensitizers, decrease hepatic glucose production and reduce insulin resistance without affecting insulin secretion by the pancreas. AMPK activation is associated with the pharmacological actions of metformin (27), whereas the transcriptional activation of PPARs is involved in the effects of thiazolidinediones $(11,12)$. Although insulin sensitizers are considered as a better agent for the treatment of T2DM patients with insulin resistance, the clinical use of thiazolidinediones, particularly rosiglitazone, is currently challenged by their severe adverse effects, including hepatotoxicity, weight gain, dyslipidemia and the possible worsening of cardiovascular risk $(28,29)$. Thus, significant efforts are concentrating on the development of novel insulin sensitizers having less toxicity. Our recent study showed that the AF extract has anti-diabetic and hypolipidemic effects via the dual agonistic action on PPAR $\alpha / \gamma$. In addition, APH was identified as the active ingredient in AF that contributes to its anti-diabetic and hypolipidemic effects in $d b / d b$ mice (22). In the present study, the anti-diabetic and anti-obesity effects of AF and APH were reconfirmed in the HFD mouse model. APH potently decreased fat cell size and alleviated cholesterol abnormalities in mice fed an HFD, thereby, at least partially, preventing weight gain. All these effects of APH are extremely similar to those of AF, indicating that APH is among major active components of AF.

Our recent study showed that AF and APH possess potent plasma glucose-lowering capabilities, even though they have relatively weak effects on PPAR $\gamma$ transactivation (22), which prompted the investigation of other mechanisms of action. The tyrosine phosphorylation of IR triggers the insulin signaling pathway, which is in turn deliberately regulated by the tyrosine phosphatase,PTP1B (30-32). The overexpression of PTP1B negatively regulates the insulin signaling pathway, leading to insulin resistance, whereas the specific inhibition of PTP1B enhances insulin signaling, which may improve insulin resistance $(32,33)$. The present data show that the blood glucose-lowering effects of $\mathrm{AF}$ and $\mathrm{APH}$ are, at least in part, mediated by repressing PTP1B action. PTP1B inhibition by AF and APH led to an increase in the tyrosine phosphorylation of IR $\beta$ and resulted in the activation of the Akt signaling pathway in $\mathrm{C} 2 \mathrm{C} 12$ myotubes (Fig. 4) and 3T3L1 adipocytes (Fig. 5). Consequently, glucose uptake was significantly enhanced by AF and APH under normal and insulin-resistant conditions (Fig. 4).

In conclusion, AF has beneficial effects on glucose and lipid metabolism in the improvement of metabolic disorders by selectively activating PPAR $\alpha$ and PPAR $\gamma$ and inhibiting PTP1B. All the described effects of AF are driven, in part, by its active component, APH. Therefore, further development of $\mathrm{AF}$ and APH as anti-metabolic agents is strongly suggested to ameliorate glucose and lipid abnormalities, insulin resistance and obesity.

\section{Acknowledgements}

The present study was supported by grants from the National Research Foundation of Korea funded by the Korean Government (MSIP) (no. 2011-0030074) and the Korea Institute of Science and Technology, Republic of Korea (no. 2Z04371).

\section{References}

1. Rathmann W and Giani G: Global prevalence of diabetes: Estimates for the year 2000 and projections for 2030. Diabetes Care 27: 2568-2569, 2004.

2. Kopelman PG: Obesity as a medical problem. Nature 404: 635-643, 2000

3. Saltiel AR: New perspectives into the molecular pathogenesis and treatment of type 2 diabetes. Cell 104: 517-529, 2001.

4. Bajaj M and Defronzo RA: Metabolic and molecular basis of insulin resistance. J Nucl Cardiol 10: 311-323, 2003.

5. Pendergrass M,Bertoldo A, Bonadonna R, Nucci G, Mandarino L, Cobelli C and Defronzo RA: Muscle glucose transport and phosphorylation in type 2 diabetic, obese nondiabetic, and genetically predisposed individuals. Am J Physiol Endocrinol Metab 292: E92-E100, 2007.

6. Cusi K, Maezono K, Osman A, Pendergrass M, Patti ME, Pratipanawatr T, DeFronzo RA, Kahn CR and Mandarino LJ: Insulin resistance differentially affects the PI3-kinase- and MAP kinase-mediated signaling in human muscle. J Clin Invest 105: 311-320, 2000.

7. Groop LC, Bonadonna RC, DelPrato S, Ratheiser K, Zyck K, Ferrannini E and DeFronzo RA: Glucose and free fatty acid metabolism in non-insulin-dependent diabetes mellitus. Evidence for multiple sites of insulin resistance. J Clin Invest 84: 205-213, 1989.

8. Bays H, Mandarino L and DeFronzo RA: Role of the adipocyte, free fatty acids, and ectopic fat in pathogenesis of type 2 diabetes mellitus: Peroxisomal proliferator-activated receptor agonists provide a rational therapeutic approach. J Clin Endocrinol Metab 89: 463-478, 2004.

9. Lee $\mathrm{CH}$, Olson P and Evans RM: Minireview: Lipid metabolism, metabolic diseases, and peroxisome proliferator-activated receptors. Endocrinology 144: 2201-2207, 2003.

10. Vidal-Puig AJ, Considine RV, Jimenez-Liñan M, Werman A, Pories WJ, Caro JF and Flier JS: Peroxisome proliferator-activated receptor gene expression in human tissues. Effects of obesity, weight loss, and regulation by insulin and glucocorticoids. J Clin Invest 99: 2416-2422, 1997.

11. Lehrke $M$ and Lazar MA: The many faces of PPARgamma. Cell 123: 993-999, 2005.

12. Evans RM, Barish GD and Wang YX: PPARs and the complex journey to obesity. Nat Med 10: 355-361, 2004.

13. Fonseca V: Effect of thiazolidinediones on body weight in patients with diabetes mellitus. Am J Med 115 (Suppl 8A): 42S-48S, 2003.

14. Bünger M, Hooiveld GJ, Kersten S and Müller M: Exploration of PPAR functions by microarray technology - a paradigm for nutrigenomics. Biochim Biophys Acta 1771: 1046-1064, 2007.

15. Bajaj M, Suraamornkul S, Hardies LJ, Glass L, Musi N and DeFronzo RA: Effects of peroxisome proliferator-activated receptor (PPAR)-alpha and PPAR-gamma agonists on glucose and lipid metabolism in patients with type 2 diabetes mellitus. Diabetologia 50: 1723-1731, 2007.

16. Pickavance LC, Brand CL, Wassermann K and Wilding JP: The dual PPARalpha/gamma agonist, ragaglitazar, improves insulin sensitivity and metabolic profile equally with pioglitazone in diabetic and dietary obese ZDF rats. Br J Pharmacol 144: 308-316, 2005.

17. Reifel-Miller A, Otto K, Hawkins E, Barr R, Bensch WR, Bull C, Dana S, Klausing K, Martin JA, Rafaeloff-Phail R, et al: A peroxisome proliferator-activated receptor alpha/gamma dual agonist with a unique in vitro profile and potent glucose and lipid effects in rodent models of type 2 diabetes and dyslipidemia. Mol Endocrinol 19: 1593-1605, 2005.

18. Harrity T, Farrelly D, Tieman A, Chu C, Kunselman L, Gu L, Ponticiello R, Cap M, Qu F, Shao C, et al: Muraglitazar, a novel dual (alpha/gamma) peroxisome proliferator-activated receptor activator, improves diabetes and other metabolic abnormalities and preserves beta-cell function in $d b / d b$ mice. Diabetes 55: 240-248, 2006. 
19. MittraS,SangleG,Tandon R,SharmaS,RoyS,Khanna V,Gupta A Sattigeri J, Sharma L, Priyadarsiny P, et al: Increase in weight induced by muraglitazar, a dual PPARalpha/gamma agonist, in $d b / d b$ mice: Adipogenesis/or oedema? Br J Pharmacol 150: 480-487, 2007.

20. Adeghate E, Adem A, Hasan MY, Tekes K and Kalasz H: Medicinal chemistry and actions of dual and pan PPAR modulators. Open Med Chem J 5 (Suppl 2): 93-98, 2011.

21. Kim T, Lee W, Jeong KH, Song JH, Park SH, Choi P, Kim SN, Lee $S$ and Ham J: Total synthesis and dual PPAR $\alpha / \gamma$ agonist effects of amorphastilbol and its synthetic derivatives. Bioorg Med Chem Lett 22: 4122-4126, 2012.

22. Lee W, Ham J, Kwon HC, Kim YK and Kim SN: Anti-diabetic effect of amorphastilbol through PPAR $\alpha / \gamma$ dual activation in $d b / d b$ mice. Biochem Biophys Res Commun 432: 73-79, 2013.

23. Matsuda J, Hosoda K, Itoh H, Son C, Doi K, Hanaoka I, Inoue $\mathrm{G}$, Nishimura H, Yoshimasa Y, Yamori Y, et al: Increased adipose expression of the uncoupling protein-3 gene by thiazolidinediones in Wistar fatty rats and in cultured adipocytes. Diabetes 47: 1809-1814, 1998.

24. Emilsson V, O'Dowd J, Wang S, Liu YL, Sennitt M, Heyman R and Cawthorne MA: The effects of rexinoids and rosiglitazone on body weight and uncoupling protein isoform expression in the Zucker fa/fa rat. Metabolism 49: 1610-1615, 2000

25. Campbell RK: Clarifying the role of incretin-based therapies in the treatment of type 2 diabetes mellitus. Clin Ther 33: 511-527, 2011.
26. Ahrén B: The future of incretin-based therapy: Novel avenues - novel targets. Diabetes Obes Metab 13 (Suppl 1): 158-166, 2011.

27. Zhou G, Myers R, Li Y, Chen Y, Shen X, Fenyk-Melody J, Wu M, Ventre J, Doebber T, Fujii N, et al: Role of AMP-activated protein kinase in mechanism of metformin action. J Clin Invest 108: 1167-1174, 2001.

28. Nissen SE and Wolski K: Effect of rosiglitazone on the risk of myocardial infarction and death from cardiovascular causes. N Engl J Med 356: 2457-2471, 2007.

29. Kermani A and Garg A: Thiazolidinedione-associated congestive heart failure and pulmonary edema. Mayo Clin Proc 78: 1088-1091, 2003.

30. Alonso A, Sasin J, Bottini N, Friedberg I, Friedberg I, Osterman A, Godzik A, Hunter T, Dixon J and Mustelin T: Protein tyrosine phosphatases in the human genome. Cell 117: 699-711, 2004.

31. Moller DE: New drug targets for type 2 diabetes and the metabolic syndrome. Nature 414: 821-827, 2001.

32. Tonks NK: Protein tyrosine phosphatases: From genes, to function, to disease. Nat Rev Mol Cell Biol 7: 833-846, 2006.

33. Elchebly M, Payette P, Michaliszyn E, Cromlish W, Collins S, Loy AL, Normandin D, Cheng A, Himms-Hagen J, Chan CC, et al: Increased insulin sensitivity and obesity resistance in mice lacking the protein tyrosine phosphatase-1B gene. Science 283: 1544-1548, 1999. 